\title{
Implementación de pruebas biométricas en sistema informático en el sector público
}

\section{Implementation of biometric testing in the computer system in the public sector}

Carlos Luis Pazmiño Palma. ${ }^{1}$, Dalia Yasmin Ortiz Reinoso. ${ }^{2}$, Byron Omar Asencio Rodríguez. ${ }^{3} \&$ Tito Hugo Ramos Soledispa. ${ }^{4}$

\section{DOI: https://doi.org/10.33262/visionariodigital.v3i3.608}

\begin{abstract}
Public institutions need an adequate control of personnel, helping them with different applications, in which Internet services are used that can be attacked in their entirety. These applications are considered of vital importance in public administration, due to the need for reports and control of human resources. The IT management of the institutions have infrastructural and access control deficiencies that generate risks and errors in the information, with economic implications. The implementation of an efficient personnel control system, considering the adequacy of the physical environment and hardware (installation of equipment and environmental safety measures), and the analysis of different technologies to implement the system is considered a priority. biometric A comparative analysis of different alternatives of biometric technologies was carried out, looking for the most reliable mechanisms for authentication in relation to the characteristics such as: reliability, security, resistance to attacks, acceptability and cost. The problem lies in the manual control of inputs, outputs and justifications, also the incomplete reports are added, which causes an inadequate decision making on the part of the organisms in charge of the control of

${ }^{1}$ Universidad: Escuela Superior Politécnica del Litoral, Magister en Sistemas de Información Gerencial, Guayaquil, Ecuador, carlos_pazmino2@ hotmail.com

2 Universidad: Escuela Superior Politécnica del Litoral, Magister en Seguridad Informática Aplicada, Guayaquil, Ecuador, dalia.ortizr@ug.edu.ec

${ }^{3}$ Universidad: Escuela Superior Politécnica del Litoral, Magister en Sistemas de Información Gerencial, Guayaquil, Ecuador, byron.asencio@gmail.com

${ }^{4}$ Universidad: Escuela Superior Politécnica del Litoral, Magister en Sistemas de Información Gerencial, Guayaquil, Ecuador, titoramos_ec@yahoo.com
\end{abstract}


the human resource, by the manual and incomplete reports, with errors and little accuracy, which does not generate any kind of validity to be so accessible to be handled by the same staff.

Keywords: Management, biometric system, security.

\section{Resumen}

Las instituciones públicas necesitan un control de personal adecuado, ayudándose para ello con diferentes aplicaciones, en las que se utilizan servicios de internet susceptibles de ser atacados en su integridad. Estas aplicaciones son consideradas de vital importancia en la administración pública, por la necesidad de reportes y control del recurso humano. La dirección de informática de las instituciones presenta falencias de infraestructura y en el control de acceso que generan riesgos y errores en la información, con implicaciones económicas. Se considerada prioritario por parte de la gestión pública, la implementación de un eficiente sistema de control de personal, contemplando la adecuación del ambiente físico y del hardware (instalación de equipos y medidas de seguridad ambientales), y el análisis de diferentes tecnologías para implementar sistema biométrico. Se realizó un análisis comparativo de diferentes alternativas de tecnologías biométricas, buscando los mecanismos más confiables para la autenticación con relación a las características tales como: fiabilidad, seguridad, resistencia a ataques, aceptabilidad y costo. El problema radica en el control manualmente de entradas, salidas y justificaciones, también se suma la realización de informes incompletos, lo que provoca una inadecuada toma de decisiones por parte de los organismos encargados del control del recurso humano, por los reportes manuales e incompletos, con errores y poca exactitud, mismo que no genera ningún tipo de validez al ser tan accesibles para ser manipulados por el mismo personal.

Palabras claves: Gestión, sistema biométrico, seguridad.

\section{Introducción.}

Las instituciones del sector público que tiene como objetivo proporcionar servicios a los ciudadanos y sus empleados para la satisfacción de sus necesidades al control y seguridad con sus respectivas áreas y funciones, el departamento de talento humano tiene un problema el registro de la asistencia en algunas instituciones hasta la actualidad de manera manual y sin el uso de tecnología y acarrea la pérdida exagerada de tiempo al realizar el control de forma manual, debido a una desactualización de técnicas informáticas, dificulta los procesos no garantiza la seguridad y se puede tomar decisiones erróneas. Consecuentemente este procedimiento tiene como deficiencia en cuanto a la pérdida de datos e información, deterioro 
de registros, archivos o expedientes, imprecisiones en el registro de información, además es tedioso realizar los informes o reportes por etapas, tiempo, personas, etc. (Filian, 2018).

Dado al no contar con un sistema automatizado con la tecnología biométrica, es ahí donde se presentan los problemas o dificultades, el problema de este control de asistencia no brinda la seguridad de que registre su asistencia en la hora de entrada, salida o días libres. Este control de asistencia "tradicional", también resulta no tan efectivo cuando el jefe (fa) del departamento de talento humano, efectúa el informe, ya sea quincenal, mensual o bimestral, etc. Por qué puede ocurrir perdida de la lista, falta de lucidez de las marcas de asistencia/inasistencia y olvido del registro de asistencia diaria.

Los reportes generados manualmente no conllevan sanciones, no generan la información necesaria, propician errores y poca exactitud que termina en un informe sin ningún tipo de validez porque todos los empleados tienen acceso a las hojas de control, manipulando la información receptada diariamente. En base a estos fundamentos, es necesario contar con una aplicación para controlar dichos procesos, de tal manera que guarde toda la información necesaria, genere reportes exactos y reales previa a cualquier sanción que se aplicará al empleado que incumpla las reglas establecidas en las instituciones.

"El método biométrico es un sistema tecnológico que usa información sobre una persona (u otro organismo biológico) para identificar a esa persona. Un sistema biométrico implicará la ejecución de datos a través de algoritmos para un resultado particular, generalmente relacionado con una identificación positiva" (Chuqui, 2014).

Para la implementación sistemas biométricos de identificación de personas, se debe realizar el estudio de factibilidad y su implementación en instituciones que no posean, a través de las direcciones de informática, a través de su personal, se encargará de la adecuación del ambiente físico y del hardware en analizar las diferentes tecnologías de control de acceso a utilizar, tareas como instalación de equipos y medidas de seguridad ambientales (control de incendios, control de temperatura, etc.). La realización en la comparación entre dos tecnologías de control de acceso biométrico, un relevamiento y un diagnóstico de las dificultades de seguridad que se presentan en el personal que accede al sector de servidores. Uno de ellos es un sistema de reconocimiento biométrico basado en la geometría de la mano y, la otra alternativa es un sistema de autenticación basado en dos modalidades biométricas: huella, iris y rostro. Por esto, es importante emplear sistemas de reconocimiento biométricos para identificar a las personas y de esta manera reforzar la seguridad de la institución.

El funcionamiento del sistema biométrico se debe tomar algunas consideraciones para su almacenamiento y la autenticación puede utilizar algoritmo y que puede tomar en consideración. "Para hacer la autentificación biométrica, primero hay que registrar a las personas que van a llevar a cabo su registro. Para el reconocimiento se usa la tecnología 
biométrica para identificar el atributo físico o de conducta elegido, en la autentificación posterior se ejecuta cuando la persona presenta su propiedad corporal o muestra su comportamiento delante un dispositivo biométrico" (Susan, 2015).

Obtener el dato biométrico elegido (dedo, mano, ojo, voz, etc.) con la ayuda de un dispositivo de adquisición, procesar los datos obtenidos con un proceso de extracción y asignación de datos biométricos y analizar el registro procesado en un repositorio local central en un token portátil. Como se aprecia en la figura 1.

Figura 1. Almacenamiento del registro biométrico

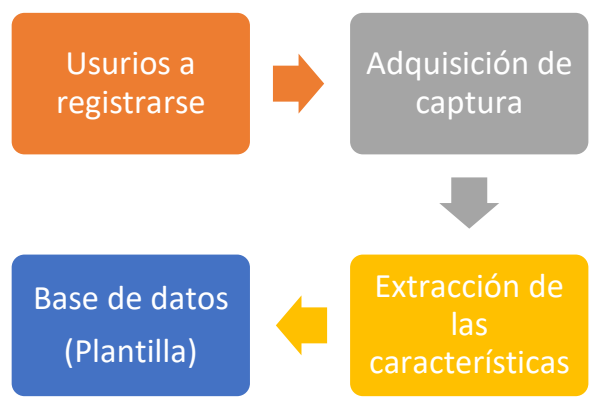

Fuente: Susan, 2015

La verificación de la identidad de un individuo se utiliza el proceso mostrado en la figura 2. Obtener el elemento biométrico elegido (dedo, mano, ojo, voz, etc.). procesar los datos biométricos y extraer el registro biométrico, verificar la conciencia del registro biométrico escaneado con los registros biométricos almacenados, mediante la utilización de los métodos estadísticos y asignar una puntuación de conciencia mediante la comparación de plantillas almacenadas y la plantilla adquirida con el dispositivo biométrico, con la finalidad de efectuar la toma de decisión e indicar si es el usuario quien dice ser.

Figura 2. Identificación y autentificación del usuario

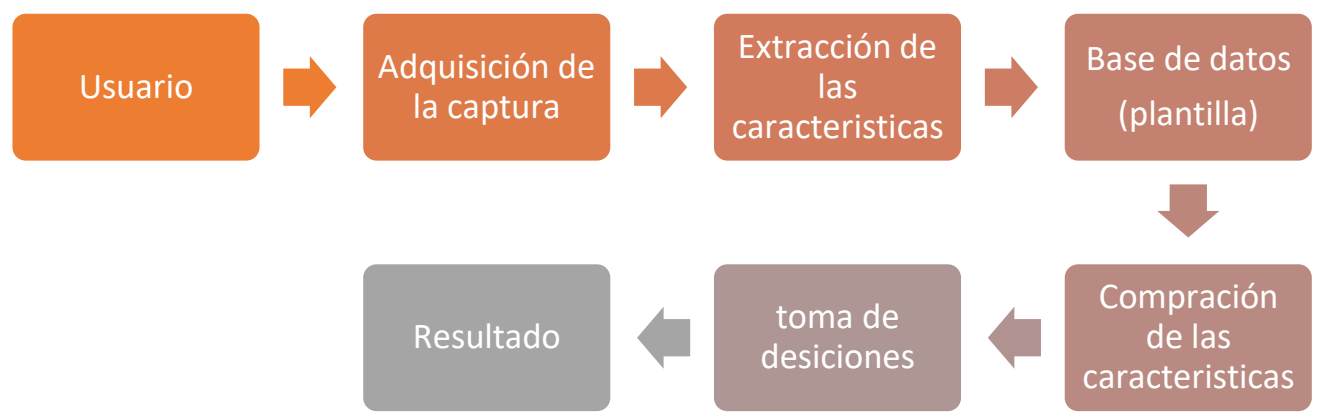

Fuente: Susan, 2015 
Los requerimientos de un sistema biométrico debe ser cien por cien confiable y debe ser universal (tomar la característica de un individuo), Unicidad (probabilidad de que dos individuos posean características muy bajas), permanencia (La característica que no cambiar a corto plazo) y la cuantificación (puede ser medido en forma cuantitativa)

\section{Análisis de requerimiento funcional}

- RF01 Reconocimiento del iris: El sistema deberá tomar una fotografía y así procesar la información adquirida.

- RF02 Identificación: el sistema deberá buscar al usuario en la base de datos.

- RF03 Validación: Se procederá a verificar si se encuentra registrado en el sistema.

- Análisis de requerimiento no funcional

\section{Los usuarios deberán identificarse para acceder al sistema.}

- El usuario visualizara solo aquellas opciones que su perfil le permite acceder.

- Los usuarios no podrán modificar su registro de entrada y salida.

El desarrollo tecnológico e innovación justifica los cambios que deben tomar en cuentas las instituciones públicas que es muy importante implementar este sistema para automatizar todos los procesos que venían llevando para el control de asistencia, ya que de esta manera se facilitará el sistema de control, lograra satisfacer al $100 \%$ los procesos de una manera rápida, ordenada y eficaz de forma global en toda la Institución para su automatización. La base es el desarrollo de pruebas biométricas, la que encierra un conjunto de actividades y procesos, que, desarrollados secuencialmente de una forma eficiente y planificada correctamente con la autoridad, que busca como principal objetivo, crear un sistema de asistencia que satisfaga las necesidades de las instituciones con un software de calidad (Arteaga \& Lasio, 2013).

De acuerdo con lo anteriormente expuesto se puede deducir claramente la imperiosa necesidad de implementar este sistema, lo que implica a la total atención al manejo de las reglas y procesos con la agilidad control y pertinencia de la información se verá reflejada en la velocidad de respuesta que se obtenga a través de la interface de salida del sistema. El proceso de automatización ayudará a que toda la operatividad de la entidad se halle enmarcada dentro de los estándares, rapidez, seguridad, solides de manejo de información y dar soluciones, mantener la satisfacción del personal.

Objetivo General: Implementar pruebas biométricas como una herramienta de control de personal para la optimización de sistema informático de las instituciones públicas a fin de controlar la asistencia de los empleados para su mejor control de personal. 
Objetivos específicos: Encontrar un marco teórico referencial de la bajo la aplicación de la interrelación entre los componentes de sistema, biométrico, componentes, procesos, como apoyo a la toma de decisiones del manejo de personal; Analizar las características del sistema biométrico para la aplicación de las instituciones públicas y Realizar las respectivas pruebas con datos reales

\section{Marco Teórico}

Marco Legal de Ecuador

\section{LEY DE PROPIEDAD INTELECTUAL SECCION V DE LOS ORDENADORES DE COMPUTADORAS}

Art. 8. La protección del derecho de autor recae sobre todas las obras del ingenio, en el ámbito literario o artístico, cualquiera que sea su género, forma de expresión, mérito o finalidad. Los derechos reconocidos por el presente Título son independientes de la propiedad del objeto material en el cual está incorporada la obra y su goce o ejercicio no están supeditados al requisito del registro o al cumplimiento de cualquier otra formalidad.

Las obras protegidas comprenden, entre otras, las siguientes:

Libros, folletos, impresos, epistolarios, artículos, novelas, cuentos, poemas, crónicas, críticas, ensayos, misivas, guiones para teatro, cinematografía, televisión, conferencias, discursos, lecciones, sermones, alegatos en derecho, memorias y otras obras de similar naturaleza, expresadas en cualquier forma; Colecciones de obras, tales como antologías o compilaciones y bases de datos de toda clase, que por la selección o disposición de las materias constituyan creaciones intelectuales, sin perjuicio de los derechos de autor que subsistan sobre los materiales o datos;

Proyectos, planos, maquetas y diseños de obras arquitectónicas y de ingeniería; lustraciones, gráficos, mapas y diseños relativos a la geografía, la topografía, y en general a la ciencia; Programas de ordenador; y, Adaptaciones, traducciones, arreglos, revisiones, actualizaciones y anotaciones; compendios, resúmenes y extractos; y, otras transformaciones de una obra, realizadas con expresa autorización de los autores de las obras originales, y sin perjuicio de sus derechos.

\section{Control de gestión}

El control de gestión es un proceso que sirve para guiar la gestión empresarial hacia los objetivos de la organización, es decir el control debe servir de modelo para alcanzar eficazmente los objetivos planteados con el mejor uso de los recursos disponibles (técnicos, 
humanos, financieros, etc.). Por lo anterior, se define como un proceso de retroalimentación de información de uso eficiente de los recursos disponibles de una empresa a fin de lograr los objetivos planteados (Barona \& Rivera, 2013)

El control de gestión asegura la economía, efectividad y eficiencia de la administración de una empresa, está en constante evaluación de los procesos internos, y monitorea a través de indicadores que denota los resultados de la gestión tanto operativa, táctica como estratégica.

\section{Viabilidad Política, Técnica y Financiera}

La Dirección Política de las instituciones públicas deben reconocer la importancia de mantener los servicios en línea de manera confiable, como requisito indispensable para el sostenimiento de los aspectos económicos, se consideró recomendar opciones de equipamiento que se detalla:

a) HANDPUNCH 1000: Lector de geometría de la mano, con capacidad para el registro de 512 usuarios y 5120 transacciones en memoria. Permite el reconocimiento 1 a 1 (verificación), y cuenta con proveedor y servicio técnico;

b) ZKSOFTWARE IFACE 202: Equipo de reconocimiento de huella dactilar y facial, con capacidad de 700 rostros, 3000 huellas dactilares y hasta 100.000 fichajes de capacidad autónoma. Permite el reconocimiento 1 a 1 (verificación), como 1 (identificación). También permite el uso de tarjeta de proximidad.

Todo dependerá del presupuestado del equipamiento de las instituciones, con relación a su costo y su crédito para cubrir la inversión.

\section{Metodología.}

Para efecto de la presente investigación se consideró un enfoque mixto cualitativo y cuantitativo, el primer enfoque detalla la aplicabilidad del sistema biométrico su descripción que permitirá evidenciar el problema y una alternativa de solución. El segundo enfoque es cuantitativo, porque la investigación se realiza y se fundamenta en datos estadísticos que parten de un estudio de campo permitiendo formar una base de datos orientados al resultado, necesario para demostrar la problemática y avizorar una solución práctica.

La metodología utilizada en el desarrollo del presente artículo se basa en el estudio descriptivo transversal, en la cual se recolecto información en un tiempo determinado, que posteriormente se tabulo y se evaluó los resultados obtenidos, permitieron identificar datos relevantes la utilización de los sistemas en prueba, permitiendo identificar los factores que influyen en la iniciativa de implementación. Las encuestas se realizaron por internet, por 
ISSN: 2602-8506

Vol. 3, N³., p. 49-62, julio - septiembre, 2019

cuanto la investigación aborda a 9 Instituciones públicas repartidas en 4 provincias, y de no hacerlo de esta manera, se elevarían los costos de manera cuantiosa, no obstante, se aprovechó a la base de datos que tienen con indicadores para el organismo de control.

\section{Resultados.}

Tabla 1. Elementos de la encuesta

\begin{tabular}{|c|c|c|c|}
\hline \multirow[t]{6}{*}{ Elementos } & Categorías & Contenido & Porcentaje \\
\hline & $\begin{array}{l}\text { Datos } \\
\text { generales }\end{array}$ & Genero & \\
\hline & & Edad & \\
\hline & & Etnia & \\
\hline & & Elección de carrera & \\
\hline & & Ocupación actual & \\
\hline \multirow{6}{*}{$\begin{array}{l}\text { Factores } \\
\text { influyentes }\end{array}$} & Condiciones & Mejorar ingresos & $67.74 \%$ \\
\hline & & Independencia Laboral & $18.45 \%$ \\
\hline & & Oportunidad de negocio & $6.45 \%$ \\
\hline & & Profesión/Vocación & $4.03 \%$ \\
\hline & & Desempleo & $2.42 \%$ \\
\hline & & Tradición familiar & $0.81 \%$ \\
\hline \multirow{5}{*}{$\begin{array}{l}\text { Factores } \\
\text { limitantes para } \\
\text { Implementar }\end{array}$} & $\begin{array}{l}\text { Condiciones } \\
\text { del entorno }\end{array}$ & Financiamiento & $49.19 \%$ \\
\hline & & Condiciones del Estado & $20.16 \%$ \\
\hline & & Entorno económico & $14.52 \%$ \\
\hline & & Temor a fracasar & $8.87 \%$ \\
\hline & & Formación del sistema & $7.26 \%$ \\
\hline \multirow{5}{*}{$\begin{array}{l}\text { Fortalezas para } \\
\text { implementar }\end{array}$} & & Elaboración de informes & $63 \%$ \\
\hline & & Preparación para el control & $62 \%$ \\
\hline & & Asesoría y seguimiento & $60 \%$ \\
\hline & & Asesoría en control & $56 \%$ \\
\hline & & Generación de redes de contactos & $46 \%$ \\
\hline
\end{tabular}


ISSN: 2602-8506

Vol. 3, N³., p. 49-62, julio - septiembre, 2019

\begin{tabular}{lll}
\hline & Acceso información & $40 \%$ \\
\hline Aporte practico & Mejor control & $47 \%$ \\
& Interacción con el personal & $43 \%$ \\
& Inserción laboral & $33 \%$ \\
\hline $\begin{array}{l}\text { Aporte teórico y } \\
\text { técnico }\end{array}$ & Necesidades del personal & $64 \%$ \\
& Contabilidad en pagos & $58 \%$ \\
& Horarios & $56 \%$ \\
& Control de horas & $51 \%$ \\
& Manejo de justificaciones & $48 \%$ \\
\hline $\begin{array}{l}\text { Aspectos que } \\
\text { sostenibilidad y } \\
\text { rentabilidad }\end{array}$ & Asistencias & $47 \%$ \\
& Innovación & $85 \%$ \\
& Generar valor económico & $72 \%$ \\
& Políticas de mejora continua y calidad & $67 \%$ \\
& Responsabilidad social & $62 \%$ \\
& Inversiones significativas & $56 \%$ \\
& Gestión de la empresa familiar & $45 \%$ \\
\hline
\end{tabular}

\section{Fuente: Encuesta}

Según Bartesaghi et al. (2016) "el intentar conceptualizar un determinado modelo determinado es difícil". Mientras que Bakhtiari (2016) menciona "el sistema es un fenómeno multicausal en el que interviene características personales de cada institución". La iniciativa emprendedora es una oportunidad de cubrir necesidades o la autorrealización en base al entorno o medio donde el emprendedor/institución se desarrolla en la búsqueda y el descubrimiento de la oportunidad (Gómez \& Manoj, 2017).

\section{DISCUSIÓN}

Factores influyentes: Mediante un enfoque integral se plantea el siguiente análisis: en la investigación realizada, la edad comprendida de las personas que pueden manejar el sistema está entre 25 y 35 años representando el 66,8\% del total encuestado. El 67,74\% de las personas estuvieron motivados por mejorar los ingresos económicos en Ecuador. Según la Comisión Económica para América Latina y el Caribe (CEPAL, 2015) los países en vías de desarrollo surgen por la necesidad, mientras que en países desarrollados se dan por 
oportunidad. Ecuador es uno de los países con mayor generación de oportunidades continente.

Fortalezas para implementar: Las instituciones el 49,19\% considera el financiamiento como un obstáculo, Según Marulanda \& Morales (2016). En América Latina y el Caribe el apoyo de los gobiernos de turno, con fuentes de financiamiento es muy limitada. Según Medrano \& Espinoza (2014). En Ecuador acceder a un crédito bancario requiere de muchos trámites burocráticos que desmotiva acceder al mismo de las instituciones. El gobierno con las entidades financieras debe establecer políticas gubernamentales que faciliten la iniciativa en la implementación de nuevos sistemas innovadores de control de personal.

Aporte practico: Las instituciones consideran importante la implementación tiene un mejor control en el 47\%, de un de un sistema biométrico que ayude como control al personal, que oriente y asesore la consecución de los objetivos institucionales, según los autores Marulanda \& Morales (2016), manifiesta que necesita asesoría/capacitación.

Aporte teórico - técnico: Las necesidades de personal en 647\% de encuestados menciona que es importante la aplicación teórica-practica de un sistema biométrico. Según los autores Blanco, López, López, \& Paz (2013) la relación con las empresas públicas y privadas genera pagos del 58\% práctica y 56\% horarios (Rodríguez, Uldarico, \& Ramírez, 2014).

Aspectos que influyen en la sostenibilidad y rentabilidad: El $85 \%$ de profesionales que laboran en las instituciones públicas, requieren sistema de innovación, Guaipatin \& Schwartz (2014) el 20.6\% de los ecuatorianos innovaron en productos y el 30\% innovo en procesos. Según este análisis comparativo el Ecuador está en el último lugar, después de Chile y Perú. Frente a lo cual el gobierno ecuatoriano invirtió 1.88\% del Producto Interno Bruto (PIB) representando alrededor de \$ 1.900 millones en la Secretaría de Educación Superior, Ciencia, Tecnología e Innovación (Senescyt).

\section{CONCLUSIONES}

1. Según los autores Román, Monsalve, \& Saravia (2016) "La sociedad reclama de las Instituciones públicas un papel más activo en el desarrollo y crecimiento económico". En este trabajo, se ha identificado que el $67.74 \%$ de los profesionales que mejorar sus ingresos. 
2. Los factores limitantes para implementar sistemas biométricos, el 49,19\% encuentra el financiamiento como un obstáculo. Según Andrade (2012) las instituciones que no tiene la posibilidad económica.

3. Las necesidades de personal que requieren las instituciones en un $64 \%$ de profesionales consideran de mucha importancia en el aporte de su contenido.

4. El $85 \%$ de los profesionales encuestados manifiestan que los sistemas deben ser innovadores, el $72 \%$ considera la importancia de crear generar valor económico,

\section{REFERENCIAS}

Álvarez, J., Romero, A., \& Murillo, D. (2014). Estrategia para la formación de competencias profesionales en la carrera de Administración de Empresas de la UNIANDES. Dialnet, 1-16.

Andrade, X. (2012). Las tablas de emprendimiento en Ecuador. Redalyc, 1-12.

Arteaga, M., \& Lasio, V. (2013). Empresas dinámicas en Ecuador: factores de éxito y competencias de sus fundadores. Redalyc, 1-12.

Bakhtiari, S. (2016). The economic sociology of new venture creation. Redalyc, 1-14.

Barona, B., \& Rivera, J. (2013). Financiación de nuevas empresas: Comparación de las fuentes de financiación en Colombia y Chile. Redalyc, 1-27.

Bartesaghi, A., SiSouza, S., Lasio, M., Varela, R., Veiga, L., Kew, P., \& Herrington, M. (2016). GEM América Latina y el Caribe. Redalyc.

Blanco, F., López, A., López, M., \& Paz, C. (2013). Las prácticas preprofesionales como herramienta de inserción laboral. Redalyc, 1-9.

CEPAL. (2015). Estudio Económico de América Latina y el Caribe. Chile: Copyright Naciones Unidas.

Chuqui, C. L. (2014). Diseño e implementación de un sistema de control de asistencia de personal. Quito: Escuela Politécnica Nacional.

Filian, M. C. (2018). Análisis de viabilidad para la implementación de un sistema de control biométrico. Los ríos: Universidad Técnica de Babahoyo. 
García, A., De la Cruz, M., García, M., Martín, J., \& Suárez, S. (2014). ¿LOS TÍTULOS DE ADMINISTRACIÓN DE EMPRESAS ADAPTADOS AL EEES EN ESPAÑA FORMAN DIRECTIVOS EMPRENDEDORES? Dialnet, 1-20.

Gómez, E., \& Manoj, C. (2017). Socio-cultural factors and youth entrepreneurship in rural regions. Redalyc, 1-20.

Guaipatin, C., \& Schwartz, L. (2014). Análisis del sistema nacional de innovación Ecuador. Quito: BID.

Hidalgo, L. (2015). La Cultura del Emprendimiento y su Formación. Dialnet, 1-5.

Lasio, V., Caicedo, G., Ordeñana, X., \& Zambrano, J. (21 de mayo de 2016). Global Entrepreneurship Monitor Ecuador. Quito: ESPAE.

Maluk, O. (2014). Variables explicativas de la intención emprendedora de los estudiantes universitarios y la importancia de la materia emprendimiento como un factor. Dialnet, 1-13.

Marín, I. (2014). Revisión teórica y propuesta de estudio sobre el emprendimiento social y la innovación tecnológica. Redalyc, 1-12.

Marulanda, F., \& Morales, S. (2016). Entorno y motivaciones para emprender. Dialnet (11), 1-13.

Medrano, E., \& Espinoza, E. (2014). Módulos auto instructivos mediante el modelo de íconos verbales en el aprendizaje de la estadística inferencial de estudiantes universitarios. Dialnet, 1-10.

OCDE. (2017). Perspectivas económicas de América Latina 2017 juventud, competencias y emprendimiento. Copyright: OECD.

Osorio, F., \& Pereira, F. (2013). Hacia un modelo de educación para el emprendimiento: Una mirada desde la teoría social cognitiva. Redalyc, 1-23.

Pereira, F., Matiz, F., García, G., \& Gómez, L. (2016). Actividad Empresarial Colombiana. Reporte GEM Colombia 2016/2017. Dialnet.

Pons, E., Torras, M., \& Hernández, X. (2015). Del modelo del evento emprendedor al modelo sistémico de emprender. Dialnet, 1-12.

Rodríguez, I., Uldarico, A., \& Ramírez, A. (2014). Apoyo al emprendimiento: De la teoría a la práctica. Redalyc, 1-17. 
Román, K., Monsalve, F., \& Saravia, J. (2016). Factores que impulsan el emprendimiento de un estudiante en la ciudad de Concepción. Dialnet, 1-18.

Sung, P., \& Duarte, M. (2015). El perfil del emprendedor y los estudios relacionados a los emprendedores Iberoamericanos. Scielo, 1-24.

Susan, K. L. (2015). Avances en técnicas biométricas y sus aplicaciones de seguridad. Carabobo: Universidad de Carabobo.

Valencia, A., Cadavid, L., Ríos, D., \& Awad, G. (2012). Factores que inciden en las intenciones emprendedoras de los estudiantes. Dialnet, 1-18.

World Bank Group. (2016). Doing Busuness 2016 Measuring Regulatory Quality and Efficiency. Redalyc, 99.

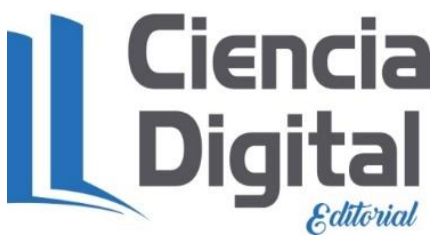




\section{PARA CITAR EL ARTÍCULO INDEXADO.}

Pazmiño Palma, C. L., Ortiz Reinoso, D. Y., Asencio Rodríguez, B. O., \& Ramos Soledispa, T. H. (2019). Implementación de pruebas biométricas en sistema informático en el sector público. Visionario Digital, 3(3), 49-62. https://doi.org/10.33262/visionariodigital.v3i3.608

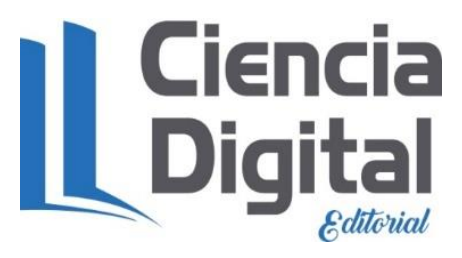

El artículo que se publica es de exclusiva responsabilidad de los autores y no necesariamente reflejan el pensamiento de la Revista Visionario Digital.

El artículo queda en propiedad de la revista y, por tanto, su publicación parcial y/o total en otro medio tiene que ser autorizado por el director de la Revista Visionario Digital.
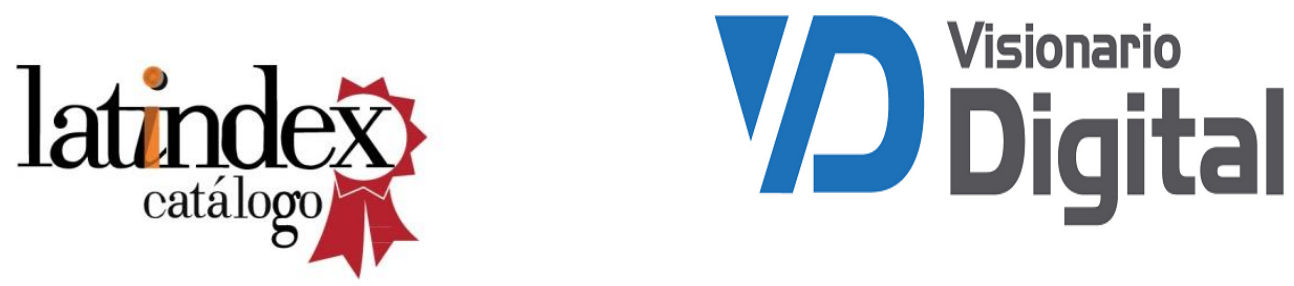